\title{
Liens entre croissance et paramètres hydriques chez les nœuds axillaires d'Hevea brasiliensis en phase d'introduction in vitro
}

\author{
H Sallanon

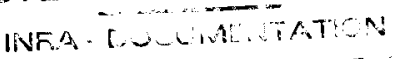 \\ 17, Rus E

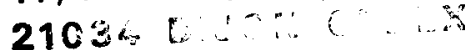 \\ Université Blaise Pascal, Laboratoire de phytomorphogenèse, 4 rue Ledru, 63038 ClermqutrFexreng. Fraweo
}

MINISTËRE DE I:'AGRICULTURE

(Reçu le 11 mai 1990; accepté le 7 juillet 1991)

\begin{abstract}
Résumé - L'augmentation du poids de matière sèche des vitroplants d'Hevea brasiliensis présente 3 phases distinctes : une phase de latence du $1^{\mathrm{er}}$ au $16^{\mathrm{e}} \mathrm{j}$ de culture, pendant laquelle l'atmosphère au-dessus des plants est proche de la saturation; puis une phase de croissance rapide, du $16^{e}$ au $30^{e} \mathrm{j}$ de culture et une phase de croissance lente du $30^{\mathrm{e}}$ au $80^{\mathrm{e}} \mathrm{j}$ pendant lesquelles l'atmosphère est plus sèche. Le suivi de l'évolution de l'état hydrique des plants et du milieu gélosé permet d'émettre l'hypothèse suivante : du $1^{\mathrm{er}}$ au $16^{\mathrm{e}} \mathrm{j}$ de culture, les plants ne peuvent pas prélever de l'eau dans le milieu gélosé; lorsque l'atmosphère est plus sèche, le flux d'eau entre plant et atmosphère favorise l'entrée d'eau du milieu gélosé dans les plants jusqu'au $30^{e} \mathrm{j}$ de culture. Au-delà, l'évaporation à l'intérieur du bocal a asséché suffisamment le milieu gélosé pour que les plants présentent les symptômes de plants hydriquement stressés.
\end{abstract}

eau / micropropagation / Hevea

Summary - Relations between growth and water parameters in axillary nodes of in vitro cultures of Hevea brasiliensis. The increase in weight of dry matter in in vitro Hevea brasiliensis is divided into 3 stages: a latency stage from the first to the 16th d of culture, during which the atmosphere inside the growth vessels is near saturation; then a fast growth stage from the :6th to the 30th d of culture, and an irregular growth from the 30th to the 80th day during which the atmosphere is drier (fig 1). The plant and medium water status variation (figs 2 and 3) led us to propose the following hypothesis: from the first to the 16th day, plants could not take up water from the medium; as the atmosphere became drier, the water flux between plant and atmosphere favoured water uptake in the plants until the 30th day. Afterwards, evaporation inside the vessel dried out the medium and plants presented symptoms of water stress.

water / micropropagation / Hevea

\section{INTRODUCTION}

Les conditions de culture in vitro sont différentes des conditions naturelles : l'humidité de l'air à l'intérieur des récipients de culture est très élevée, l'éclairement et la photosynthèse sont faibles. Les vitroplants étant essentiellement hétérotrophes (Fournioux et Bessi, 1986; Pospisilova et al, 1988; Schoch et al, 1989), le milieu nutritif contient d'importantes quantités de sucre. Ceci confère à ce milieu un potentiel hydrique plus faible que celui des solutions hydroponiques couramment utilisées. Les gradients de potentiel hydrique entre le milieu et les plants sont faibles ou inexistants (Pospisilova et al, 1988), et les forces de rétention de l'eau dans le milieu gélosé sont un des facteurs environnementaux souvent cités pour leurs influences sur le développement des plants (Debergh et al, 1981; Kozai et al, 1986). Plus récemment, des mesures du déficit de pression de vapeur (DPV) de l'atmosphère interne des récipients ont permis de mettre en évidence que le développement des plants est tout aussi dépendant des variations de l'humidité de l'atmosphère des récipients que du potentiel hydrique du milieu gélosé (Sallanon et al, 1989; Sallanon et Coudret, 1990). Ces mêmes auteurs ont montré que de très faibles variations du déficit de pression de vapeur (DPV) de l'atmosphère (20-40 $\mathrm{Pa})$ sont suffisantes pour provoquer des modifications importantes de la croissance et de la morphogenèse des vitroplants (Sallanon et al, 1989). II ressort de ces travaux que la croissance et le développement des plants en culture in vitro sont fortement dépendants de leur état et de leur environnement hydriques. 
Les protocoles d'introduction en culture in vitro d'Hevea brasiliensis sont différents de ceux traditionnellement utilisés, notamment en ce qui concerne la fermeture des récipients de culture, principal paramètre dont dépend la valeur de l'humidité à l'intérieur des récipients. Afin de déterminer si les conditions de culture ont une influence sur l'état hydrique des plants d'Hevea, l'évolution de l'état hydrique et de la croissance de ces plants sera étudiée au cours de la phase d'introduction.

\section{MATÉRIEL ET MÉTHODES}

Le matériel végétal utilisé, Hevea brasiliensis, est cultivé par la Société de microbouturage de l'hévéa et par l'Institut de recherche sur le caoutchouc à Montpellier. L'explant provient de pieds mères juvéniles issus de graines et correspond à un segment de tige portant un bourgeon axillaire. D'après le protocole établi par Enjalric et Carron (1982), les morceaux de tige sont rincés à l'éthanol $(70 \%)$ et désinfectés à l'eau oxygénée (55 VP) pendant $15 \mathrm{~min}$. Trois rinçages à l'eau distillée suivent la désinfection, les explants sont alors trempés dans une solution d'induction au débourrement et transférés sur un milieu d'expression du débourrement dépourvu de régulateur de croissance. Après $16 \mathrm{j}$ de culture en tubes, les explants sont transférés sur un milieu neuf en bocal non hermétique pendant $64 \mathrm{j}$. Afin de caractériser le type de fermeture des récipients, les pertes d'eau dues à la non étanchéité ont été évaluées par pesées successives. Ces mesures ont été réalisées sur 15 récipients qui contiennent seulement du milieu gélosé et sur 15 récipients qui contiennent en plus des plants. En $16 \mathrm{j}$ de culture en tubes, les pertes de poids sont de 3,5 \pm $0,5 \mathrm{~g}$ et de $3,1 \pm 1 \mathrm{~g}$ respectivement pour des tubes avec ou sans plants. Ensuite, pendant les 64 j de culture en bocaux, elles sont de $43 \pm 8 \mathrm{~g}$ pour des bocaux qui contiennent des plants et de $40 \pm 8 \mathrm{~g}$ pour des bocaux qui contiennent seulement du milieu gélosé. II est intéressant de noter qu'il existe une variabilité importante dans la fermeture des récipients. La température et le déficit de pression de vapeur des chambres de culture sont respectivement de $28^{\circ} \mathrm{C}$ et de $2700 \pm 700 \mathrm{~Pa}$; l'éclairement est de $100 \mu \mathrm{mol}$. $\mathrm{m}^{-2} \cdot \mathrm{s}^{-1}$ et la photopériode est de $12 \mathrm{~h} / 24 \mathrm{~h}$.

Les paramètres mesurés sont la teneur en eau : $T E=100(P M F-P M S) / P M S$, la teneur en eau relative : TER $=100(P M F-P M S) /(P F S-P M S$ ) (où $P M F=$ Poids de matière fraîche, $P M S=$ poids de matière sèche, $P F S$ = poids de matière fraîche à saturation) et le potentiel hydrique ( $\psi$ ). Les mesures de potentiel hydrique des plants et du milieu gélosé sont effectuées à l'aide d'un hygromètre à point de rosée WESCOR HP 115. Les sondes hygrométriques ont été étalonnées préalablement avec des solutions de $\mathrm{NaCl}$ de potentiels hydriques connus. Les échantillons végétaux sont les plants entiers (entre le $1^{\text {er }}$ et le $30^{\mathrm{e}} \mathrm{j}$ de culture); à partir du $40^{\circ} \mathrm{j}$, les mesures sont effectuées séparément sur la souche et sur le jeune rameau. Le potentiel hydrique du milieu gélosé est déterminé sur des cylindres de $1 \mathrm{~cm}$ de diamètre et de hauteur, situés sous les plants. La réalisation des mesures nécessite un temps d'équilibration d'environ $2 \mathrm{~h}$, à $25^{\circ} \mathrm{C}$.

II a été procédé à 5 répétitions pour chaque mesure. En raison du faible nombre de répétitions, le test U de Mann et Whitney (Schwartz, 1963) a été utilisé pour déterminer si 2 groupes de valeurs sont significativement différents à une date donnée. Pour donner une indication de la dispersion, chaque moyenne de 5 mesures est accompagnée de l'écart type.

\section{RÉSULTATS}

La croissance des plants présente 3 périodes distinctes : 1 phase de latence pendant laquelle le poids de matière sèche ne varie pas entre le $1^{\text {er }}$ et le $18^{\mathrm{e}} \mathrm{j}$, suivie d'une phase de croissance rapide entre le $18^{\mathrm{e}}$ et le $30^{\mathrm{e}} \mathrm{j}$, et d'une phase de croissance plus irrégulière entre le $30^{\circ}$ et le $80^{\circ} j$ de culture (fig $1 \mathrm{~A}$ ). Le poids de matière fraîche des explants augmente régulièrement entre le $1^{\text {er }}$ et le $5^{\mathrm{e}} \mathrm{j}$, puis se stabilise jusqu'au $18^{\mathrm{e}} \mathrm{j}$ de culture (fig 1B). Il augmente ensuite chez les explants âgés de 18 à $30 \mathrm{j}$ (fig 1B). Entre le $30^{\circ}$ et le $70^{\mathrm{e}} \mathrm{j}$, le poids de matière fraîche ne varie pas; une augmentation significative n'est observée qu'entre le $70^{\circ}$ et le $80^{\circ}$ j de culture (fig 1B). La teneur en eau reste constante chez les explants âgés de 1 à $30 \mathrm{j}$. La teneur en eau de la souche diminue ensuite jusqu'à la fin de la culture; celle du rameau diminue jusqu'au $60^{\circ} \mathrm{j}$, puis se stabilise (fig 2A). Les poids de matière sèche et fraîche et les teneurs en eau et en eau relative de la souche sont supérieurs à ceux du rameau (figs 1 et 2).

Le potentiel hydrique des plants varie peu durant les 30 premiers j de culture, sa valeur est d'environ $-1,5 \mathrm{MPa}$ (fig $2 \mathrm{C}$ ). II diminue ensuite progressivement jusqu'à $-2 /-3 \mathrm{MPa}$. Cette valeur minimale est atteinte le $60^{\mathrm{e}} \mathrm{j}$ de culture; le $80^{\mathrm{e}} \mathrm{j}$ les plants ont un potentiel hydrique de $-1,5$ à $-2 \mathrm{MPa}$. Le potentiel hydrique de la souche est toujours sensiblement supérieur à celui du rameau (fig $2 \mathrm{C}$ ).

Le potentiel hydrique du milieu gélosé ne varie pas durant les 30 premiers $j$ de culture; il diminue ensuite progressivement de $-0,6$ à $-1,5 \mathrm{MPa}$ entre les $30^{\circ}$ et $80^{\circ} \mathrm{j}$ de culture (fig 3 ). 

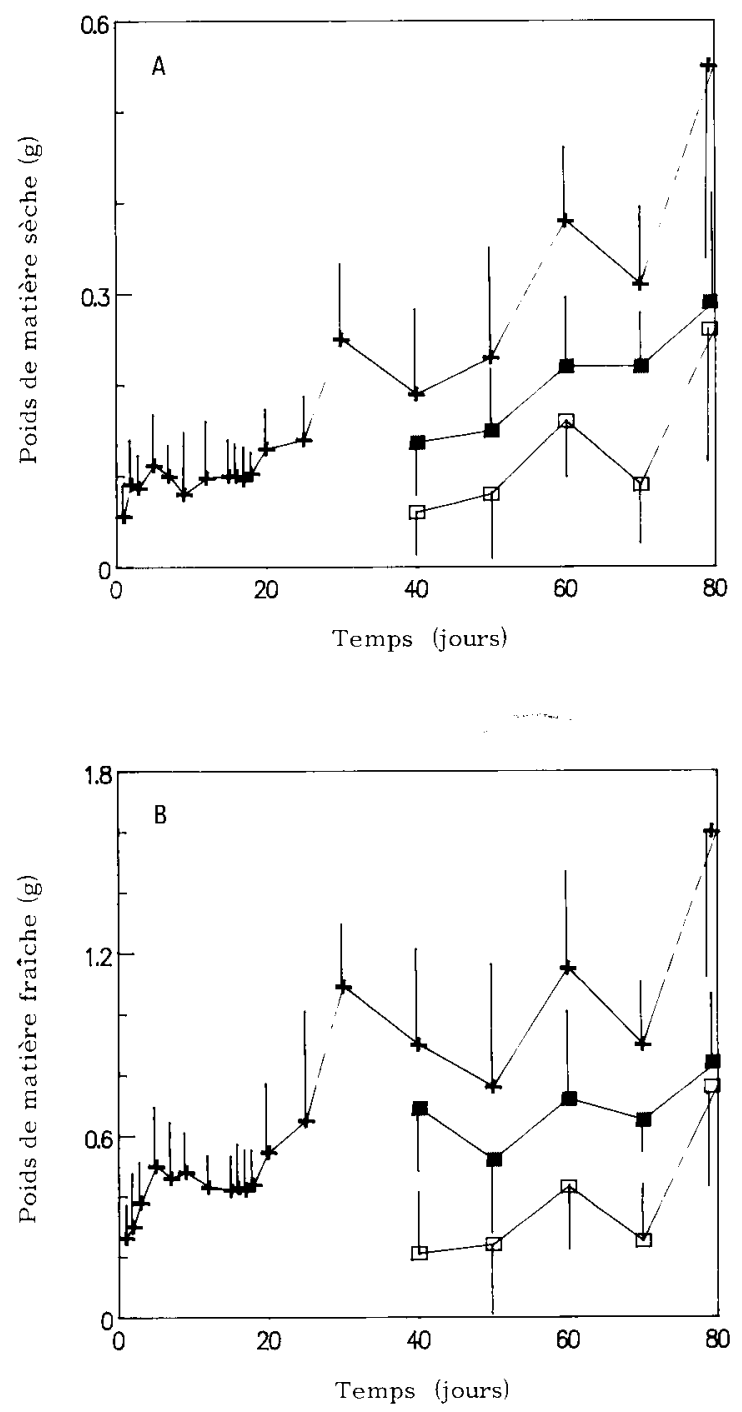

Fig 1. Évolution du poids de matière sèche (PMS) (A), et du poids de matière fraîche (PMF) (B) (en g), en fonction du temps (en j); (1) : plante entière; (2) : souche; (3) : rameau. Chaque résultat est une moyenne de 5 mesures encadrée de l'écart type. $+1 ;--2 ;-\square-3$.

\section{DISCUSSION}

Pendant toute la durée de la culture en tubes (les 16 premiers $\mathrm{j}$ ), le poids de matière sèche reste constant et le poids de matière fraîche n'augmente que pendant les 5 premiers $\mathrm{j}$ de culture. Un enrichissement en eau des plants durant cette période n'est observé que pendant les $5 \mathrm{j}$ qui suivent la mise en culture. L'eau qui pénètre dans les plants peut avoir théoriquement 3 origines différentes. :

- le milieu gélosé;

- une mince couche d'eau qui se dépose sur le milieu gélosé et provient du ruissellement de
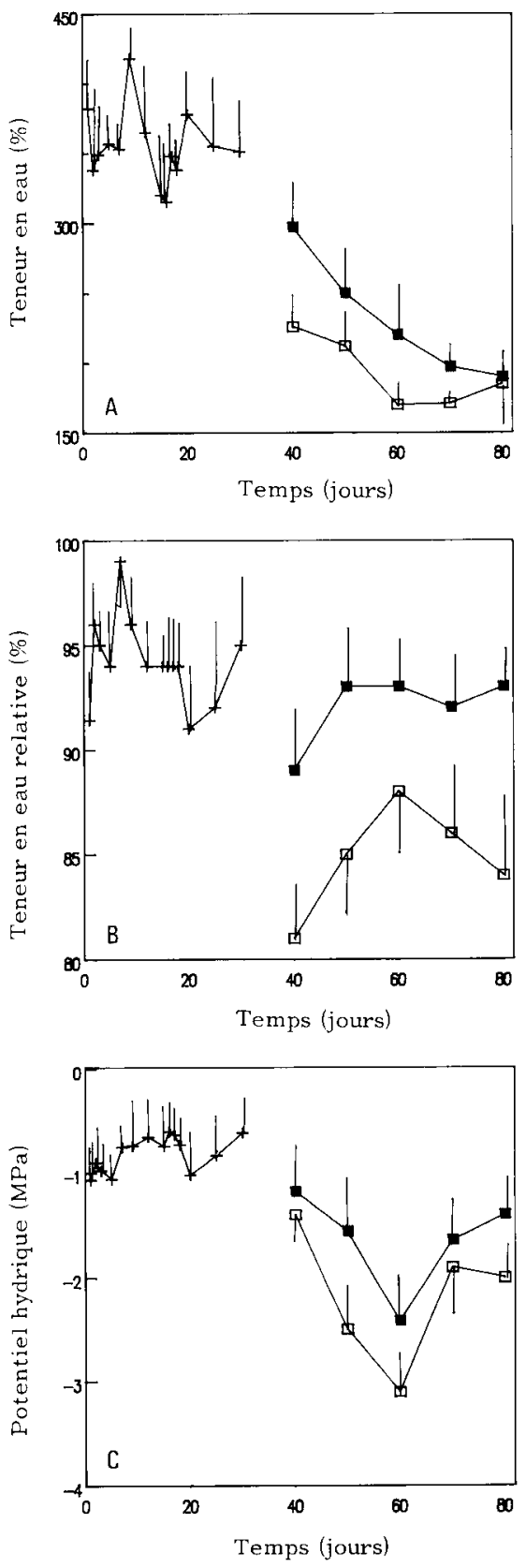

Fig 2. Evolution de la teneur en eau (A) (en \%) de la teneur en eau relative (B) (en \%), et du potentiel hydrique (C) (en $\mathrm{MPa}$ ) en fonction du temps (en j); (1) : plante entière; (2) : souche; (3) : rameau. Chaque résultat est une moyenne de 5 mesures encadrée de l'écart type. $+1 ;--2 ;-\square-3$.

l'eau condensée sur les parois des récipients de culture, immédiatement après autoclavage;

- l'eau atmosphérique.

Afin d'apprécier quelles sont les origines les plus probables de cette eau, les potentiels hydriques de chacune de ces formes d'eau ont été mesurés :

- le potentiel hydrique du milieu gélosé est peu différent de celui des plants; 


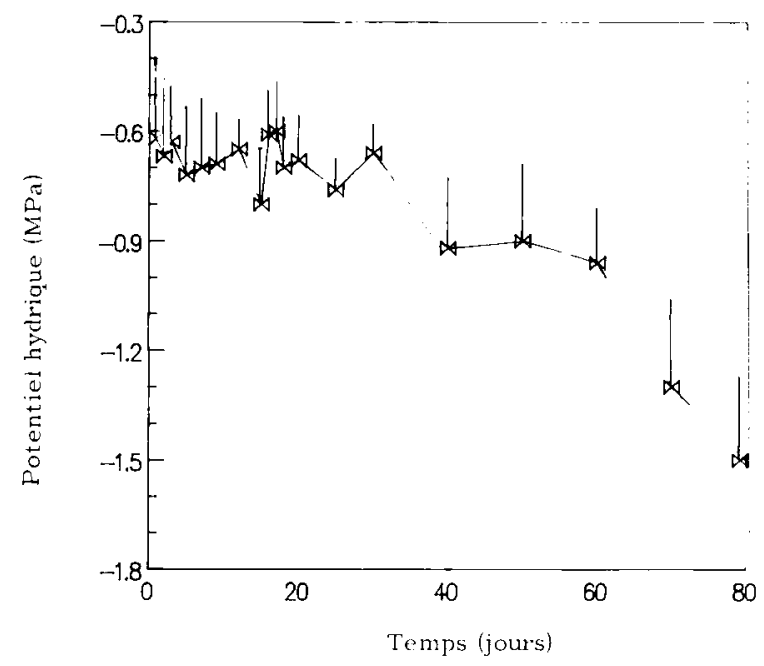

Fig 3. Évolution du potentiel hydrique du milieu gélosé (en $\mathrm{MPa}$ ) en fonction du temps (en j). Chaque résultat est une moyenne de 5 mesures encadrée de l'écart type.

- l'eau qui se dépose sur le milieu gélosé a un potentiel hydrique égal à celui du milieu nutritif liquide avant l'apport d'agar, et supérieur d'environ $-0,1 \mathrm{MPa}$ à celui du milieu nutritif gélosé;

- le déficit de pression de vapeur (DPV) de l'atmosphère, déterminé à l'aide d'un hygromètre à point de rosée est voisin de 0 pendant les 16 premiers j de culture.

Bien qu'en terme de potentiel hydrique, l'eau atmosphérique soit la plus libre, le fait qu'une pénétration d'eau dans les plants n'ait lieu que pendant les 5 premiers $j$ de culture laisse supposer que l'eau d'hydratation des plants provient de la pellicule de milieu liquide présent à la surface du milieu gélosé : cette eau, en effet, disparaît rapidement quelques $\mathrm{j}$ après l'autoclavage, par évaporation, par incorporation dans le milieu gélosé, ou par absorption par les plants.

À partir du $5 \mathrm{e} j$ de culture, les poids de matière fraîche et sèche ainsi que les paramètres hydriques mesurés restent stables et ce, jusqu'au $16^{\mathrm{e}} \mathrm{j}$ de culture. Durant cette période, les échanges hydriques entre le milieu, les plants et l'atmosphère, peuvent être de 2 natures différentes :

- l'eau absorbée est entièrement perdue par transpiration;

- les échanges milieu-plants et plantsatmosphère sont très faibles ou inexistants.

Pour définir la nature de ces échanges, il est nécessaire de connaître quels sont les processus qui interviennent dans l'établissement de l'humidité à l'intérieur des récipients de culture. Cette humidité varie avec :

- la fuite de vapeur d'eau vers l'extérieur au niveau du couvercle;

- l'évaporation à partir du milieu gélosé et des plants;

- la condensation de l'eau sur les parois des récipients et sur le contenu du bocal, puis son évaporation (ceci étant lié aux différences existant entre la température de l'air et celles de la paroi et du contenu du bocal).

Si l'intensité de l'évapotranspiration est constante, le $D P V$ à l'intérieur des récipients augmente avec l'intensité de la fuite de vapeur d'eau. Lorsque cette dernière ne varie pas, le $D P V$ à l'intérieur du récipient diminue avec l'augmentation de l'évapotranspiration. Si la fuite et l'émission de vapeur d'eau varient, les valeurs d'équilibre du $D P V$ seront d'autant plus faibles que la fuite sera faible et que l'évapotranspiration sera importante (Sallanon, 1990).

Au cours de la culture en tubes, l'intensité de la fuite est constante; l'atmosphère à l'intérieur des tubes étant quasi saturée en eau, la fuite de vapeur d'eau doit être relativement faible par rapport à l'émission de vapeur d'eau. Si la fuite était nulle (récipients hermétiquement fermés), et la température parfaitement stable et homogène, l'équilibre entre les concentrations en vapeur d'eau dans l'atmosphère et dans le contenu du bocal conduirait à des valeurs de $D P V$ de l'atmosphère supérieures aux valeurs déterminées expérimentalement. L'obtention de ces valeurs expérimentales nécessite un accroissement de l'émission de vapeur d'eau qui peut être dû à l'évaporation de l'eau condensée sur les parois des tubes. En présence d'une atmosphère aussi humide, de très faibles écarts de température suffisent pour provoquer la condensation de l'eau. La saturation de l'atmosphère à l'intérieur des récipients de culture ne peut pas être due aux pertes d'eau par évapotranspiration, mais proviendrait de l'équilibre entre la condensation et l'évaporation de l'eau condensée sur les parois des tubes.

Dans une telle atmosphère, la transpiration doit être faible et l'hypothèse selon laquelle les échanges milieu-explant et plants-atmosphère sont très faibles ou inexistants paraît la plus probable.

Une pénétration d'eau dans les plants ne redevient effective que lorsque le poids de matière fraîche augmente, à partir du $18^{\mathrm{e}} \mathrm{j}$ de culture, date à laquelle est également observé le début 
de la croissance. Les plants âgés de $18 \mathrm{j}$ sont identiques aux plants âgés de $16 \mathrm{j}$, la taille du jeune rameau varie entre 0,5 et $1,5 \mathrm{~cm}$ de hauteur et le plant ne possède pas de racines. Ces plants ont été transférés en bocaux dont le système de fermeture permet des fuites de vapeur d'eau vers l'extérieur plus importantes que pendant les 16 premiers $\mathrm{j}$ de culture. Les fuites de vapeur d'eau étant ainsi augmentées, le $D P V$ de l'air à l'intérieur des bocaux devient plus important. Les mesures expérimentales montrent qu'il est supérieur ou égal à $200 \mathrm{~Pa}$. L'évapotranspiration, contrôlée par la différence de pression de vapeur dans l'air et à la surface du milieu gélosé et des plants, augmente également. D'autre part, une nouvelle pellicule de milieu liquide s'est formée à la surface du milieu gélosé récemment autoclavé. Ce sont ces modifications environnementales qui permettent probablement une absorption d'eau et le début de croissance des plants observés à partir du $18^{\mathrm{e}} \mathrm{j}$ de culture.

Au-delà du $40^{\mathrm{e}} \mathrm{j}$ de culture, les plants présentent des symptômes de plants hydriquement stressés : la teneur en eau et le potentiel hydrique diminuent. Ceci coîncide avec la diminution du potentiel hydrique du milieu gélosé. Les pertes d'eau cumulées au cours du temps conduisent à un assèchement du milieu gélosé, qui en réduisant l'évaporation, participe à l'augmentation du $D P V$ de l'air à l'intérieur des bocaux et donc du pouvoir évaporant. L'état de stress hydrique qui affecte les plants est dû à une diminution de la disponibilité en eau dans le milieu gélosé et à une augmentation du pouvoir évaporant de l'atmosphère. D'autre part, le passage de l'eau du milieu gélosé vers le rameau peut être rendu difficile par la structure de la souche (formation de cals à chaque extrémité), ou par la croissance de la souche elle-même, croissance accompagnant celle du rameau. La souche détournerait alors à son profit l'eau et les substances nutritives; le rameau toujours moins hydraté que cette dernière, limiterait ses pertes en eau et photosynthétiserait : Lardet (1987) a montré que les rameaux photosynthétisent plus qu'ils ne respirent à partir du $20^{\mathrm{e}} \mathrm{j}$ de culture.

Durant cette période un grand nombre de feuilles se nécrosent, tombent, et des racines se développent à partir de la souche. Ce développement racinaire facilite l'approvisionnement en eau des plants et peut expliquer la faible reprise de croissance observée pendant les 10 derniers j de culture. La coexistence d'une croissance et d'un état de stress hydrique prononcé est d'ailleurs assez fréquemment décrite (Steudle et al, 1982; Bozarth et al, 1987).

\section{CONCLUSION}

La croissance des vitroplants d'Hevea est conditionnée par leurs possibilités d'approvisionnement en eau, lesquelles dépendent essentiellement du type de bouchage des récipients de culture. Les relations hydriques entre l'explant et son environnement ne sont satisfaisantes qu'entre le $16^{e}$ et le $40^{e}$ j de culture. En deça, les échanges milieu-explant sont très faibles et au-delà, une évapotranspiration forte conduit à un assèchement du milieu gélosé et place les plants en état de stress hydrique. Une augmentation des échanges gazeux entre les atmosphères interne et externe des récipients de culture pourrait faciliter l'absorption d'eau par les plants en début de culture. Une diminution des échanges gazeux avec l'extérieur ou une augmentation du potentiel hydrique du milieu gélosé, à partir du $16^{\mathrm{e}} \mathrm{j}$ de culture, sont proposées pour éviter l'état de stress rapidement atteint.

\section{REMERCIEMENTS}

Je remercie vivement MP Carron, responsable du laboratoire de culture in vitro de l'Institut de recherche sur le caoutchouc, pour la fourniture et les informations concernant la croissance des plants d'Hevea, sans lesquelles ce travail n'aurait pas pu être réalisé.

\section{RÉFÉRENCES}

Bozarth CS, Mullet JE, Boyer JS (1987) Cell wall proteins at low water potentials. Plant Physiol 85, 261267

Debergh P, Harbaoui Y, Lemeur R (1981) Mass propagation of globe artichoke (Cyanara scolymus) : evaluation of different hypotheses to overcome vitrification with special reference to water potential. Physiol Plant 53, 181-187

Enjalric F, Carron MP (1982) Microbouturage in vitro de jeunes plants d'Hevea brasiliensis (Kunth) MüllArg. CR Séances Acad Sci 295, 259-264

Fournioux JC, Bessi R (1986) Influence de la teneur en gaz carbonique sur la morphogenèse de la vigne en culture in vitro. Can J Bot 64, 2608-2616

Kozai T, Fujiwara K, Watanabe I (1986) Fundamental studies on environments in plant tissue culture vessels (1) Relations between the culture medium 
composition and water potential of liquid culture media. J Agric Meteorol 42, 1-6

Lardet $L$ (1987) Amélioration des conditions du microbouturage de l'Hevea brasiliensis Müll-Arg. Thèse Doct Université des Sciences et Techniques du Languedoc, $242 \mathrm{p}$

Pospisilova J, Solarova J, Catski J, Ondrej M, Opatrny $Z$ (1988) The photosynthetic caracteristics during the micropropagation of tobacco and potato plants. Photosynthetica 22, 205-213

Sallanon H (1990) Caractérisation de l'environnement hydrique en culture in vitro. Relation avec l'état hydrique et la morphogenèse chez Rosa hybrida variété deladel et Hevea Brasiliensis Muel-Arg. Thèse Doct Université Clermont-Ferrand, $110 \mathrm{p}$
Sallanon H, Coudret A (1990) Flux d'eau entre vitroplants et atmosphère en micropropagation. $C R$ Acad Sci 310, 607-613

Sallanon H, Mazière Y, Brenckmann F (1989) Effects of air relative humidity and of agar concentration on water status and growth of roses micropropagation. Colloque Moët Hennessy, Aix-les-Bains

Schoch PG, Lefebre B, Tesson C, Ganry J (1989) Photosynthèse et respiration chez le bananier in vitro. Photosynthetica 23, 113-118

Schwartz D (1963) Méthodes statistiques à l'usage des médecins et des biologistes. Flamarion, Paris, $235 p$

Steudle E, Zimmermann U, Zillikens J (1982) Effect of cell turgor on hydraulic conductivity and elastic modulus of Elodea leaf cells. Planta 154, 371-380 